\title{
Las fuentes de imputación de la Responsabilidad penal corporativa*
}

\author{
Raquel Roso Cañadillas
}

Profesora Titular de Derecho Penal

Universidad de Alcalá, Madrid

raquel.roso@uah.es

\section{Resumen}

La LO 5/2010, aprobada el 22 de junio y que entró en vigor el pasado 23 de diciembre de 2010 contiene dos fuentes de imputación penal de la persona jurídica. Este trabajo se centra en el análisis de estas fuentes y ofrece una construcción interpretativa del precepto, contribuyendo a delimitar su ámbito de aplicación.

\section{Palabras clave}

Fuente de imputación / provecho / falta de vigilancia y de control / personas físicas comprometedoras

* Se incluye dentro del Proyecto de Investigación titulado Protección penal del sistema económico, empresarial y laboral en el marco de los mercados, cuya referencia es Der 2008-02004/JURI y financiado por el Ministerio de Ciencia e Innovación. 


\section{Abstract}

The Law 5/2010, passed the 22nd June, and that has come into effect the past December the 23th of 2010, contains two different sources of attribution of criminal liability to legal persons. The present work focuses on the analysis of those sources, offering an interpretative construction of the law, and helps to interpret the scope of the law.

\section{Key words}

Source of attribution of liability / benefit / lack of supervision and control / individual incriminating persons

\section{Tabla de contenido}

1. Introducción. 2. Fuentes de imputación de la responsabilidad penal de las personas jurídicas: Responsabilidad penal corporativa. 3. Cuestiones de dolo $o$ imprudencia en las fuentes de imputación. 4. Consecuencias y objetivos de la Reforma en este ámbito. Referencias bibliográficas 


\section{Introducción}

1. Desde el día 23 de diciembre de 2010 la persona jurídica tiene responsabilidad penal en el ordenamiento jurídico-penal español, produciéndose uno de los cambios de más calado de los últimos tiempos en la concepción del Derecho Penal y del destinatario de la norma. Esta revolución de paradigmas empezó un 27 de noviembre de 2009, cuando el Congreso publicó el Proyecto de ley que modificaba la LO 10/1995. Meses después, el 28 de abril de 2010, tras el Dictamen de la Comisión de Justicia, el pleno del Congreso aprueba por mayoría absoluta el texto del Proyecto. E1 Senado lo hará después. Finalmente la Reforma se publica el pasado 22 de junio de 2010 con una vacatio de seis meses, entrando en vigor el pasado mes de diciembre.

En este punto, como reitero, la reforma es revolucionaria, marcará un antes y un después en el Derecho penal y en el proceso penal, ya que de tener como centro de imputación penal a la persona física, se pasa a una bicefalia: persona física y persona jurídica. La implantación de este sistema con toda probabilidad va a dar lugar a una posterior labor de retoques y de ajustes sustanciales y procesales, pero el cambio ya se ha producido y habrá que acatarlo y aplicarlo, pese a la marcada controversia dogmática que ha habido y hay sobre la responsabilidad penal de las personas jurídicas.

2. Antes de entrar en el desarrollo de la cuestión concreta a la que dedico las siguientes páginas, quisiera realizar alguna aclaración terminológica, sobre todo, en cuanto al concepto de persona jurídica.

El CP no hace en ninguno de sus artículos una descripción de lo que se debe entender por persona jurídica a efectos penales y un sector doctrinal considera que habrá que adoptar el concepto extrapenal de persona jurídica, lo que encuentro a estos efectos perfectamente asumible. La Reforma, por el contrario, lo que sí hace es contribuir a perfilar este concepto por la técnica de la exclusión y así excluye:

a) Al Estado, a las Administraciones públicas territoriales e institucionales, a los Organismos Reguladores, las Agencias y Entidades Públicas Empresariales, a los partidos políticos y sindicatos, a las organizaciones internacionales de derecho público, y a aquellas otras que ejerzan potestades públicas de soberanía, administrativas o cuando se trate de Sociedades mercantiles Estatales que ejecuten políticas públicas o presten servicios de interés económico general (art. 31 bis 5, primer inciso $\mathrm{CP}$ ). 
No obstante, ¡excepción!, se podrá declarar la responsabilidad penal de este tipo de Organismos, Agencias, Entidades, si no corresponden a lo que dicen que son y se utilizan entonces instrumentalmente estas formas para eludir cualquier eventual responsabilidad penal como persona jurídica (art, 31 bis, 5, segundo inciso $\mathrm{CP}$ ).

b) Y también se excluyen del art. 31 bis todas aquellas empresas, organizaciones, grupos o cualquier otra clase de entidades o agrupaciones de personas a las que, por carecer de personalidad jurídica, no se les pueden aplicar las reglas de responsabilidad penal establecidas en el art. 31 bis del CP para las personas jurídicas, en cuyo caso se reservan las consecuencias accesorias. (art. 129).

En conclusión tenemos una primera clasificación de entidades con personalidad jurídica a las que se le imponen penas y entidades sin personalidad jurídica a las que se le imponen consecuencias accesorias.

También podemos encontrarnos con otra clasificación no positivizada expresamente, pero sí implícitamente como ahora veremos, y que se ubica ya dentro del grupo de las entidades con personalidad jurídica. Atendiendo al nivel de criminalización de la persona jurídica y a su instrumentalización podemos distinguir entre personas jurídicas de actividad lícita relevante y que corresponde con su objeto social y otras personas jurídicas de actividad ilícita relevante, pese a que su constitución inicial obedece a un objeto social lícito, con el que se ha creado una falacia de legalidad y. sin embargo, esconden tras de sí toda una infraestructura dedicada al delito.

Las personas jurídicas de actividad lícita se suelen corresponder con la idea de empresa, ya que ésta se forma con el propósito exclusivo de poner en marcha una actividad lícita y que además genere un beneficio económico y social. Los delitos cometidos por este tipo de personas jurídicas: empresas, lo son con ocasión del desarrollo de su actividad, lícita, que en momentos puntuales puede deslizarse hacia la ilicitud. Sin embargo, la actividad legal desarrollada por las personas jurídicas de actividad ilícita relevante no son más que operaciones metódicamente planeadas y al servicio de las operaciones ilícitas, que son el verdadero objetivo de estas empresas. También se puede presentar la posibilidad de que, si tomamos como referente el histórico de la compañía o corporación, ésta se iniciase en una actividad lícita y finalmente termine cultivando constante y continuamente actividades ilícitas, convirtiéndose así en su objeto y razón de ser. Sea como fuere, al ordenamiento 
jurídico no le importan en este caso tanto los inicios como los finales y así efectivamente en el art. 66 bis se puede encontrar cómo la ley toma como criterios la reincidencia y la relevancia de la actividad ilícita dentro de la empresa para imponer las penas interdictivas e incluso la disolución del art. 33. 7. Por último, hay que señalar que incluso dentro de la lista cerrada de delitos seleccionados encontramos una tipología de delitos relativos al tráfico de órganos, la trata de seres humanos, la prostitución y corrupción de menores, la financiación de terrorismo o los ataques a la salud pública, que parecen estar pensados correlativamente para la persona jurídica con actividad relevantemente ilícita.

\section{Fuentes de imputación de la responsabilidad penal de las personas jurídicas: Responsabilidad penal corporativa}

El núcleo de la regulación reside en diseñar las fuentes de imputación que justifican la imputación penal y correlativamente la responsabilidad penal de las personas jurídicas. La Reforma ha seleccionado y fijado dos fuentes de responsabilidad: el provecho y la falta de control, o descontrol. Efectivamente el art. 31 bis 1.del Código Penal dice que la persona jurídica será responsable:

a) cuando o los representantes legales o los administradores de hecho o de derecho, actúen, cometiendo uno de los delitos previstos expresamente, en nombre o por cuenta de la misma y obtengan un provecho para la persona jurídica.

b) cuando personas físicas, sometidas a la autoridad de los representantes legales, o los administradores de hecho o de derecho, han podido cometer los hechos delictivos, en el ejercicio de actividades sociales y por cuenta y provecho de la persona jurídica, por no haberse ejercido sobre ellas el debido control atendidas las concretas circunstancias del caso.

I. La primera fuente, el provecho, está huérfana de cualquier descripción legal, lo que dará lugar a un desarrollo interpretativo que favorecerá la casuística. En cualquier caso, es obvio que el provecho tiene que repercutir inexorablemente en la corporación, aunque también se beneficie el administrador o representante, pero lo que no puede ser objeto de responsabilidad penal de la persona jurídica es la exclusiva consecución de beneficio individual, sin beneficio corporativo. El beneficio, para un sector doctrinal con el que estoy de acuerdo, puede ser económico o de otra naturaleza, puede tener una plasmación directa en el balance económico de la empresa o una repercusión indirecta por ahorro de costes. 
Pero lo que más puede preocupar es el exceso de objetividad con el que se ha definido esta fuente. La ley ha focalizado toda la justificación de la imputación en la producción de un resultado: el provecho, pero éste tiene que venir acompañado necesariamente de un desvalor de la acción y de una responsabilidad subjetiva o desvalor de la intención del sujeto activo, de lo contrario la regulación no habría respetado las bases esenciales y creo que inmutables del Derecho Penal. No cabe otra alternativa que pensar que la ley ha dado todo esto por sobreentendido. Y ello es así, porque, por un lado, el precepto se ubica dentro de un sistema y con base en él debe hacerse su interpretación y completarlo. Y por otro lado, no hay que perder de vista que sólo es posible en Derecho penal castigar el provecho cuando éste es ilicítamente obtenido y esto sólo se consigue no por la sola obtención del provecho, sino por la forma en la que se ha obtenido ese provecho. Precisamente a la obtención del provecho es a lo que se dedican la mayoría de las sociedades mercantiles, sujetos de esta nueva regulación, y sería absurdo pensar que el Derecho penal con esta Ley ha pretendido acabar con la obtención del beneficio y con ello con el sistema económico. Por tanto, necesariamente el Derecho penal no está castigando la obtención de un provecho, sino la obtención de un provecho ilícitamente obtenido, ya que el provecho es un concepto en sí mismo positivo y lo que lo hace disvalioso es la forma de conseguirlo.

Sentado lo anterior, el provecho debe obtenerse reuniendo los siguientes requisitos. En primer lugar, el representante, administrador de hecho o de derecho tiene que realizar un delito:

1. De los expresamente previstos, se establece un sistema de numerus clausus. Por tanto, hay una lista de delitos y es una lista cerrada, en la que se encuentran delitos económicos, medioambientales, urbanísticos o tipos delictivos que castigan determinada forma de relacionarse las empresas con las administraciones públicas u organismos internacionales como el cohecho, el tráfico de influencias o la corrupción en las transacciones internacionales. Por último, encontramos el tráfico de órganos, la trata de seres humanos, la prostitución y corrupción de menores, la financiación del terrorismo o los ataques a la salud pública,

2. Ya con dolo, ya con imprudencia, aunque en este primer inciso del art. 31 bis, como más adelante propongo interpretar, se castigará más el delito doloso, siendo la imprudencia una excepción.

3. En nombre de la sociedad o por cuenta de ésta; si no lo hace en tal condición no podrá comprometer a la persona jurídica. Ahora bien, yendo más allá del propio carácter nominal y aunque la ley no lo mencione expresamente, también se puede llegar a excluir la relación de compromiso, si el hecho realizado 
por el círculo de personas físicas comprometedoras no entra dentro de las competencias que le otorga su cargo o, usando la fórmula del art. $295 \mathrm{CP}$, con abuso de las funciones de su cargo, porque nominalmente puede estar representado a la sociedad y sin embargo materialmente no estar dentro de los límites del mandato otorgado, lo que invalida el acto de representación o de gestión de la sociedad. Pero estos supuestos, son más complejos de lo que en un principio pueda parecer, porque la persona jurídica puede ser un instrumento en manos de sus representantes y en el caso concreto no tener responsabilidad penal alguna en el hecho cometido por el representante en su nombre, o también cabe la hipótesis de hacer responsable a la persona jurídica por consentir dolosamente o por imprudencia por falta de diligencia los abusos del administrador. Dependerá entonces la imputación de la persona jurídica de conseguir fundamentar la existencia de dolo o imprudencia en el abuso de competencias del representante o administrador.

En conclusión, cuando el administrador de hecho o de derecho o el representante han llevado a cabo un delito de la parte especial con todos los requisitos anteriores, la persona jurídica será responsable por dejar dolosa o imprudentemente que se obtenga un provecho ilícito. Ello es lo que se ha dado en llamar un defecto en su organización. Así la persona jurídica se conduce con falta de diligencia, cuando permite sin saberlo y debiendo haberlo sabido, o con dolo, cuando consiente a las claras, la comisión del delito por sus propios representantes y administradores.

En el fondo detrás de la fuente de responsabilidad de obtención de un provecho ilícito nos encontramos también con la falta de control por parte de la persona jurídica, pero aquí, al contrario del segundo inciso, como más adelante se expone, la falta de control se da en una relación bilateral y directa entre la persona jurídica y su cúpula directiva. De tal modo que con esta interpretación la primera fuente de responsabilidad se fundamenta en la falta de control por parte de la persona jurídica de su equipo directivo, permitiendo que éste obtenga un provecho ilícito que beneficia a la sociedad. Se está castigando o la culpa in eligiendo unida a la omisión de controles y de vigilancia de la tarea directiva y representativa o una actitud inductora o de cooperación de la persona jurídica con la persona física, que pone al servicio de ésta toda su organización y estructura para conseguir el provecho ilícito perseguido.

II. La segunda fuente de responsabilidad penal de las personas jurídicas también exige el provecho, como elemento económico, pero el elemento central que configura la fuente de imputación es la falta de control, pero ahora la falta 
del ejercicio de ese control, al que se refiere la ley expresamente, no es sobre los administradores, sino sobre los sometidos a la autoridad de esos administradores, por lo que el itinerario delictivo se dibuja de la siguiente manera: en primer lugar, el subordinado o el sometido a la autoridad del representante o administrador debe cometer alguno de los hechos típicos de la lista legal seleccionada expresamente; en segundo lugar, este subordinado no ha debido ser vigilado, ni fiscalizada su labor y por ello precisamente ha podido realizar holgadamente y sin obstáculo alguno que vencer su acción delictiva, es decir, el representante o administrador no ha ejercido sus obligaciones de debido control y diligencia sobre el subordinado; en tercer lugar, la persona jurídica es penalmente responsable, ya sea por falta de diligencia, ya sea por consentir dolosamente, por mantener al frente de su empresa representantes o administradores que incumplen el deber específico; de todos los que puedan tener, el incumplimiento de éste es el que tiene relevancia penal, el no vigilar y controlar a sus subordinados.

En este supuesto, al contrario que en el primer inciso del art. 31 bis, la relación entre los sujetos activos es triangular, es decir, deben concurrir tres planos superpuestos de responsabilidad subjetiva: debe haber responsabilidad del subordinado, del círculo de personas comprometedoras y de la persona jurídica. Pero el requisito indispensable en ambas fuentes es la falta de una autoorganización eficiente y adecuada en el seno de la persona jurídica que evite gestiones dolosas o imprudentes por parte de su equipo directivo. Se impone la era del control eficiente como barrera de contención de los riesgos delictivos.

III. Tanto en la primera fuente de responsabilidad, como en la segunda fuente de responsabilidad las personas físicas que la ley ha seleccionado han sido los representantes legales y los administradores de hecho y de derecho, dejando aparte otro tipo de proposiciones que se han barajado a lo largo de la tramitación de la Reforma. Finalmente se ha apostado por un círculo más reducido de personas físicas comprometedoras dejando afuera la figura del apoderado circunstancial o a los mandos intermedios. No obstante, pese al esfuerzo legal realizado, siempre quedan fuera supuestos o aspectos concretos de casos que plantean una nueva problemática que la ley no ha contemplado. Así las sociedades, sobre todo las grandes empresas, adoptan organizaciones complejas atendiendo a la actividad desarrollada o a las ventajas fiscales que puedan obtener y nos encontramos, por poner un ejemplo, con la cuestión de si en un holding, los administradores de la sociedad participada pueden comprometer a la sociedad inversora y arrastrarla al proceso penal. Quizás estas cuestiones debían ser tratadas en una normativa externa, que desarrollara la extensión de los deberes de control en macrosociedades por la matriz o por el holding respecto a las participadas o filiales y en qué casos, si lo hubiera, y sobre qué actividades. 


\section{Cuestiones de dolo o imprudencia en las fuentes de imputación}

3.1 Para dar respuesta a si las fuentes de responsabilidad penal de las personas jurídicas están concebidas de tal modo que cabe tanto la realización de forma dolosa como imprudente, hay que tener en cuenta la regulación de la imprudencia en nuestro CP, así como la lista cerrada de delitos que se han seleccionado por la Reforma de 2010 y que generan la responsabilidad de las personas jurídicas. Por tanto, se parte de dos criterios de restricción.

\section{Ateniéndonos al art. 12 y puesto éste en relación con los delitos seleccionados} en la parte especial para imputar a la persona jurídica, sólo se podría castigar a ésta, cuando expresamente se hubiera tipificado el delito en su modalidad imprudente. La persona jurídica sería responsable $\mathrm{p}$. ej. porque los subordinados de sus administradores han cometido un delito debido a la falta de vigilancia $\mathrm{y}$ de control que se ha ejercido sobre estos empleados, lo que demuestra a su vez que la sociedad no ha controlado a sus gestores, con lo que la persona jurídica podría ser responsable penalmente por un delito imprudente de los que están expresamente previstos. Esta posibilidad se planteará en muy pocas ocasiones, si acogemos esta interpretación de la regulación, porque viene marcada por la punición tasada del delito imprudente en la parte especial.

Esta aplicación tan mínima a la que se puede llegar del delito imprudente dentro del ámbito de responsabilidad de las personas jurídicas debe ser aplaudida, si es lo que se pretendía, tras analizar y concluir que los niveles de eficacia punitivos y la corrección, por tanto, de las autoorganizaciones corporativas se sustanciaba castigando los tipos dolosos y no tanto los imprudentes.

Sin embargo, si acudimos a la realidad podemos constatar que en el origen de muchos de los defectos de organización empresarial, el comportamiento se describe a través de una falta de diligencia. Ahora bien, ello es así si nos centramos en una sociedad o corporación con un objeto social lícito y una actividad lícita. Hay que admitir que no es el caso si la sociedad tiene como objeto y fin último la realización de hechos delictivos por sistema y en su sistema, en el que la intención y el dolo están presentes desde el momento mismo de su constitución. Pero si lo que se ha querido es concienciar y dirigir a las empresas para que implanten un modelo de organización basado en el control, el paradigma es la corporación de actividad lícita y su forma de expresión delictiva más habitual es la imprudencia. 
En una sociedad de actividad lícita, los actos corporativos o colectivos se dirigen a obtener un beneficio económico lícito, porque el procedimiento para conseguirlo se ha planteado en el ámbito de la licitud. Si el beneficio obtenido revierte en ese caso en concreto en ilícito, algo ha ocurrido en el proceso de consecución del beneficio. Y si ese "algo" no se debe a la casualidad o a lo fortuito, el siguiente escalón, al que debemos acudir, nos señala la intromisión de una conducta humana no lo suficientemente diligente. $Y$ eso es lo que ocurre en una corporación o empresa con objeto lícito que excluye a priori necesariamente dentro de su esquema de negocio la ilicitud del beneficio, por cualquier vía. En ella, no se ha querido el resultado, pero éste se ha producido por falta de cuidado o diligencia en el proceder de la persona física y de la persona jurídica.

Por otro lado, no tanto en la primera fuente, que, como hemos visto, aparentemente ha renunciado a describir el modo de actuación y se ha dirigido frontalmente a imputar por la producción del resultado, si se cumple el resto de requisitos, pero sí en la segunda de las fuentes de imputación, la forma en que el legislador ha descrito la conducta lesiva es propia más de una actuación imprudente que de una dolosa, cuando habla de que los subordinados han podido precisamente realizar el hecho por no haber ejercido sobre ellos el debido control, porque si el administrador o el representante se ha puesto de acuerdo con los subordinados e interviene, aunque sea meramente consintiendo (participación o autoría dolosa) en la realización del delito, que en la mayoría de los casos no será sólo así, para castigar al administrador o representante no sería necesaria una cláusula como la del art. 31 bis 1, inciso segundo, bastaría y sobraría con la aplicación de los preceptos que castigan la autoría o en el supuesto más benigno la participación dolosa en un hecho doloso y, ello sin olvidar que también resultaría, dentro de la complejidad, más fácil articular la responsabilidad penal de la persona jurídica.

3.2 Pero ya en el caso de la imprudencia, las cosas se complican más y es necesario según el art. 12 que una fórmula descriptiva comprendida en un precepto penal recoja expresamente la punición de la forma imprudente y éste sería el caso del inciso segundo del art. 31 bis 1 . del CP. Se puede sostener así que el art. 31 bis cumple el mandato legal del art. 12 y posibilita el castigo también por imprudencia de la persona jurídica, cuando su administrador o representante no haya ejercido el debido control sobre sus subordinados, ya haya sido con dolo, ya haya sido por imprudencia, y ello sin que en el delito concreto se haya castigado la versión imprudente. 
Recordemos que el art. 31 bis 1 . inciso segundo recoge tres niveles de superposición punitiva, ya que en el primer nivel el subordinado tiene que realizar uno de los delitos seleccionados por la regulación, en el segundo nivel tiene que probarse que una de las personas físicas comprometedoras realizó una falta del ejercicio debido de control y que además ésta contribuyó a que el subordinado delinquiese, porque de otra manera gracias al debido control se podía haber evitado, y en un tercer nivel la persona jurídica resulta imputada por mantener en sus puestos de gestión y representación un tipo de personal con graves deficiencias de formación e instrucción en su capacidad directiva.

A este esquema hay que sumarle la parte subjetiva: el dolo o la imprudencia. Así el subordinado tendrá que cometer el delito tal y como éste esté recogido en la parte especial; ello significa que si se exige dolo, sólo se castigará el delito si ha habido dolo en la conducta del empleado y la modalidad imprudente estará excluida, consecuencia que se extenderá al círculo de personas comprometedoras y a la persona jurídica. Todos quedarán impunes. Si en cambio el delito contempla tanto la modalidad imprudente como la dolosa y el subordinado actúa con imprudencia, entonces ya la responsabilidad podrá ascender pasando por la persona física comprometedora hasta llegar a la persona jurídica comprometida y ésta podría ser imputada, si se cumple el resto de requisitos.

\section{La duda se plantea en los casos en los que el tipo sólo contempla la forma} dolosa y el subordinado efectivamente ha actuado con dolo, y en cambio la persona física comprometedora lo ha hecho no ejerciendo el debido control sobre su subordinado, pero en su modalidad imprudente, es decir, con falta de diligencia actuando con desidia, con dejadez, incluso si se quiere con apatía dejando de vigilar y controlar las actividades de las personas que estaban directamente a su cargo. Aprovechando la coyuntura el subordinado delinque con voluntad y conciencia al hacerlo. En este caso, se puede sostener, como ya lo hace un sector doctrinal, que si el tipo sólo admite la forma dolosa, el art. 31 bis 1 segundo inciso no es una cláusula de extensión de la punición imprudente y no se podrá seguir ascendiendo en la cadena de imputación. Esta interpretación es, sin duda, admisible y es una solución político-criminal que no plantea problemas a la hora de aplicar las penas. Pero hay otras dos posibles soluciones si interpretamos que en la fórmula legal utilizada: no ejercer sobre ellos el debido control, cabe tanto el dolo como la imprudencia, convirtiéndose así en un supuesto de punición de la imprudencia. 
a) La primera de las soluciones apunta al castigo del superior jerárquico por haber permitido, al hacer dejadez de sus funciones, que delinca el subordinado. Una vez que se afirma la imprudencia o falta de diligencia de la persona física comprometedora, se puede subir al siguiente nivel y comprobar que la falta de diligencia del administrador o representante ha sido consentida o se ha producido por la falta de control que se debía haber ejercido a su vez sobre los administradores o representantes, siendo por ello imputada la persona jurídica.

Esta interpretación no encuentra problemas con la definición legal del art. 31 bis 1, más bien, yo diría, que al contrario. El obstáculo se encuentra a la hora de aplicar las penas, porque si no está recogida la modalidad imprudente en el tipo de la parte especial, hay que preguntar cómo castigar a la persona física y a la persona jurídica sin que salten todas las alarmas conectadas al principio de legalidad. La Reforma, por su parte, permite hacer una interpretación en la que se puede castigar la conducta imprudente, pero no parece estar refrendada por la regulación de la parte especial. Sin embargo, la realidad criminógena frente a la que parece que se quiere luchar, y vuelvo a repetir en sociedades con actividad lícita, es en su gran mayoría de perfil imprudente. Se produce entonces una distonía entre la realidad y los objetivos de la regulación.

Quizás lo menos arriesgado es replegar filas y renunciar al intento de buscar una respuesta punitiva a este tipo de imprudencias en sociedades de actividad lícita, por mucho que el art. 31 bis 1 abra una espita a la imprudencia, la cual no puede implantarse y prosperar por no verse secundada por la parte especial.

Quizás lo más arriesgado, pero que permite dar una respuesta punitiva a imprudencias, por supuesto siempre graves, cometidas dentro del seno de corporaciones de actividad lícita sea seguir abriendo esa espita todo lo que la Reforma actual permita, de tal modo que si el subordinado comete el delito doloso, porque el superior jerárquico ha permitido con su actuación imprudente que ocurra, se podría castigar al superior jerárquico comprometedor por el art. 31 bis 1 inciso segundo, en el que se tipifica la imprudencia, puesto en relación con el tipo concreto y determinar su pena conforme al límite inferior de la penal legal impuesta para el delito doloso, a falta de una pena específica y para atenernos en lo posible al principio de proporcionalidad, aunque es evidente, si no la vulneración frontal, la fricción como mínimo que se produce con el principio de legalidad. En el caso de la persona jurídica encuentro menos escrúpulos legales para castigarla por la falta de control a su vez imprudente sobre la gestión de 
sus administradores y representantes. Y ello porque en la mayoría de los casos los parámetros para determinar concretamente la pena en el caso de la persona jurídica son varios (art. 66 bis) y de muy distinta naturaleza al factor subjetivo, lo que nos demuestra que se toman en cuenta otros criterios que le son más propios a la responsabilidad penal de la persona jurídica, y el factor subjetivo, aun estando necesariamente presente, tiende a diluirse entre el resto de criterios para determinar la pena. Incidiendo en este argumento se puede sostener que la existencia de dolo o imprudencia no da lugar en el caso de la persona jurídica a penas esencialmente diferentes. Así la imposición de una pena de multa y el resto de penas interdictivas no viene dada por la existencia de dolo o imprudencia, sino por los criterios desarrollados en el art. 66 bis CP. Por tanto, a la luz de este sistema de penas y de los criterios de determinación de la pena manejados por la Reforma, considero que el elemento subjetivo tiene un espacio de aplicación distinto dentro de las penas aplicables a las personas jurídicas, como es el de servir para graduar la extensión temporal de la pena o la cuantía en cada caso. En conclusión, las penas reguladas en la parte especial para las personas jurídicas sirven indistintamente para castigar tanto un defecto de organización imprudente como doloso, sin que esta regulación así entendida menoscabe el principio de legalidad y el de proporcionalidad.

b) La segunda solución, teniendo en cuenta el problema que plantea en la solución anterior la inexistencia de una determinación concreta de la pena del tipo imprudente en la parte especial, pese a que el art. 31 bis 1 segundo inciso, se entienda como una cláusula de extensión de la imprudencia, pasaría por no castigar al superior jerárquico. Efectivamente, son de gran peso los argumentos para defender la no punibilidad del superior jerárquico, empezando porque no existe ninguna pena en la parte especial para la imprudencia, que sí que castiga la parte general en el art. 31 bis 1 . No obstante, renunciar a castigar al superior jerárquico, que es el administrador o representante de la empresa, puede suponer la interrupción de la cadena de imputación y de este modo la impunidad injusta de la persona jurídica, sobre todo en el caso de que la persona jurídica comete un defecto doloso de control o de vigilancia de su personal comprometedor.

Como vemos, el galimatías de estas responsabilidades superpuestas del art. 31 bis $1 \mathrm{CP}$ nos lleva a preguntarnos de qué clase es la interdependencia entre la persona jurídica comprometida y el círculo de personas comprometedoras, es decir entre la persona jurídica y sus personas físicas, y sobre todo la pregunta es si es necesario que la persona física haya cometido un delito para poder obtener a su vez la imputación de la persona jurídica. 
En la descripción legal recogida en las fuentes es notoria la dependencia material de la persona jurídica de la persona física, pues es responsable en la medida en que determinadas personas físicas con poder de mando y competencia dentro de la estructura empresarial y que forman parte de sus órganos de gobierno no hagan bien sus deberes. Pero esta interrelación material acaba aquí y la regulación, de manera coherente con la admisión de la responsabilidad penal de la persona jurídica, proclama una responsabilidad independiente que evita los problemas de confrontación con el non bis in f́dem. Incluso, para salvar la oposición de cuestiones de punibilidad o de procedibilidad y dejar clara la absoluta independencia, cuando no se haya individualizado a la concreta persona física que lo ha cometido, pero se sabe que pertenece al grupo de elegidos por el precepto penal, o cuando por la razón que sea no se ha podido dirigir el procedimiento penal contra la concreta persona física (art. 31.bis 2.), la persona jurídica será responsable penalmente.

Pero pese a que se proclama la autonomía de la responsabilidad penal de la persona jurídica, existe un nexo de unión intangible al que podríamos denominar principio de vinculación física. En efecto, la persona jurídica no será responsable si su persona o personas físicas no realizan una falta de control o vigilancia de sus subordinados. Y, en efecto, esa conducta delictiva atendiendo a la descripción del art. 31 bis 1 resulta constituir la prueba fehaciente de que la persona jurídica (obviamente a través de decisiones u omisiones de su presidencia o consejo de administración) a su vez no ha controlado, ni vigilado a su personal de dirección, ya sea con doloso consentimiento, ya sea por una grave falta de diligencia. Si nos atenemos entonces a lo prescrito por el art. 31 bis 1, hay que llegar a la conclusión de que la persona física comprometedora ha cometido el hecho típico descrito en este precepto, pese a que su conducta imprudente no encuentra correlativamente la pena que le corresponde en los tipos designados en la parte especial; pero a los efectos que aquí interesan se han ido realizando las actuaciones típicas diseñadas en cada nivel de responsabilidad: subordinado, representante o administrador y persona jurídica, lo que permite hacer responsable a la persona jurídica, pese a que la persona física quede sin responsabilidad penal por falta de pena, que no por atipicidad. Si por el contrario optamos por negar la responsabilidad penal de la persona jurídica cuando no se castigue a la persona física por la razón mencionada, podemos llegar a la solución de la imposibilidad de castigar a la persona jurídica en los casos en los que el subordinado actúe con dolo, la persona física comprometedora con imprudencia, sin pena en la parte especial y la persona jurídica con una actitud de doloso consentimiento ante la falta de diligencia de sus directivos. 
Pero además la persona física comprometedora es como mínimo partícipe omisivo imprudente en un hecho doloso o imprudente del subordinado, porque también esta hipótesis cabe en la descripción de la segunda fuente del art. 31, 1 bis, aunque en la parte especial el legislador se ha olvidado de regular todas estas posibilidades.

3.3 Por último, en el último momento, valga la redundancia, de su tramitación y aprobación, en la segunda de las fuentes se introdujo la coletilla de la falta del control debido en el caso concreto. Evidentemente con ello se quiere extirpar de este sistema de responsabilidad cualquier tentación de implantar un Derecho penal de autor en el seno de las personas jurídicas y castigar la conducta (o conducción) de vida y no el hecho concreto. Así quedarán fuera situaciones en las que la empresa carezca de programas preventivos o de control o de autoorganización evitando riesgos de esa actividad o de todas o de algunas, pero sin embargo se compruebe que esa falta de organización, en cualquier caso, disciplinaria nada tuvo que ver con la producción del hecho delictivo que realizó el subordinado.

3.4 Todo este desarrollo no es trasplantable a la primera de las fuentes, porque en ella no podemos encontrar una cláusula de punición expresa de la imprudencia, tal y como exige el art. $12 \mathrm{CP}$. Y por tanto, la obtención imprudente del provecho ilícitamente obtenido sólo se castigará cuando expresamente lo admita la ley, por lo que se castigará en casi todos los casos por hechos dolosos.

\section{Consecuencias y objetivos de la Reforma en este ámbito}

Como toda norma penal, la Reforma aspira a cambiar conductas y comportamientos en la sociedad, no sólo a regularlos y a ordenarlos. Pretende la concienciación y la prevención. Así ante esta perspectiva la persona física tiene que tomar conciencia de que cada vez que delinca en el seno de su persona jurídica, y lo haga en provecho de ésta y representándola o lo haga no controlando los desmanes o descuidos de sus subordinados, arrastra al juzgado con ella, también como imputada, a su persona jurídica. Y, por su parte, la persona jurídica tiene que tomar conciencia, obviamente a través de su presidencia o su directiva, de que debe implantar controles para obtener sólo el "provecho lícito" y no verse mezclada en "provechos ilícitos", de tal modo que la responsabilidad se quede en la esfera de la persona física que ha cometido el delito, sin salpicar a la persona jurídica. Un buen blindaje y protección será la articulación de un óptimo sistema de control, que, por otro lado, no ahogue la actividad económica de la empresa 
y no instaure la desconfianza dentro de la plantilla laboral, ni dentro del órgano de gobierno por las evaluaciones constantes de su trabajo o de la fiscalización de su gestión.

El Derecho penal envía ahora el mensaje nítido de que esto no puede ser de otra manera y las empresas deben evolucionar hacia un desarrollo integral del "control". Esto sólo se puede hacer a través de mecanismos de control externo, como son las auditorías y las propias reglas del mercado, y mecanismos de control interno, por medio de programas de cumplimiento, de seguimiento, formación de las personas cuyos cargos impliquen decisiones y las cuales puedan identificar los riesgos de las decisiones tomadas, sistemas de archivo de información, selección metódica de información y elaboración de memorias globales, a modo de Global Reporting (GR, reportes globales), elevadas a los Consejos de Administración u órganos de control, porque éstos deben estar informados de la realidad poliédrica de su empresa y tomar decisiones teniendo en cuenta las consecuencias globales y de conjunto, evitando cualquier tipo de riesgos y en primera línea los penales por ser los más básicos y los de mayor trascendencia para ellos mismos. Hay que remarcar que el cáncer en una empresa empieza con un Consejo de Administración desinformado, bien sea porque no se le informa, bien sea porque la información es de mala calidad, al ser el producto de un proceso de selección erróneo y desenfocado de la información. En estos casos se puede acabar en una degeneración de la actividad empresarial, que revierte en la transformación del riesgo penal en una lesión real. El poder punitivo del Estado por medio del Derecho penal acabará aplicando una multa a la empresa o hará uso de las demás penas, en un segundo escalón de intensidad punitiva, entre ellas sobre todo de la intervención judicial, que pronóstico que será un instrumento eficaz, siempre que se realice dentro de un proceso dominado por la sensatez y la honestidad, para ordenar y gestionar el conflicto sin grandes costes sociales, económicos y de desarrollo y sin deterioro del bienestar social. 